\title{
Dendritic cells matured with recombinant human sperm associated antigen 9 (rhSPAG9) induce CD4+, CD8+ $T$ cells and activate NK cells: $A$ potential candidate molecule for immunotherapy in cervical cancer
}

Hemavathi Dhandapani

CANCER INSTITUTE (WIA)

Hascitha Jayakumar

CANCER INSTITUTE (WIA)

Abirami Seetharaman

CANCER INSTITUTE (WIA)

Selvaluxmy Ganeshrajah

CANCER INSTITUTE (WIA)

Shirley Sunder Singh

CANCER INSTITUTE (WIA)

Nirmala Jagadish

NATIONAL INSTITUTE OF IMMUNOLOGY

Anil Suri

NATIONAL INSTITUT EOF IMMUNOLOGY

Rajkumar Thangarajan

CANCER INSTITUTE (WIA)

Priya Ramanathan ( $\sim$ priya.serkin@gmail.com )

Cancer Institute (WIA)

\section{Research}

Keywords: SPAG9, cisplatin, Th1, IFNy, Chemo-adjuvant therapy

Posted Date: July 28th, 2020

DOI: https://doi.org/10.21203/rs.3.rs-46780/v1

License: (c) (i) This work is licensed under a Creative Commons Attribution 4.0 International License.

Read Full License 
Version of Record: A version of this preprint was published at Cancer Cell International on September 7th, 2021. See the published version at https://doi.org/10.1186/s12935-021-01951-7. 


\section{Abstract \\ Background}

Dendritic cell (DC)-based immunotherapy is capable of activating the immune system, and in particular tumour-specific cytotoxic T lymphocytes (CTLs) to eradicate the tumour. However, major limitations are the availability of autologous tumour cells as antigenic source and the selection of antigen that may have potential to activate both CD8 + and CD $4+T$ cells in immune-specific manner. Recently, we reported the expression of sperm associated antigen 9 (SPAG9) that is associated with various types of malignancies including cervical cancer. We examined the recombinant human SPAG9 (rhSPAG9) as an antigenic source for generating efficient $D C s$ to stimulate $C D 4+$ and $C D 8+T$ cell responses for future DCs-based vaccine trials in cervical cancer patients.

\section{Methods}

Human monocytes derived DCs were pulsed with different concentrations $(250 \mathrm{ng} / \mathrm{ml}$ to $1000 \mathrm{ng} / \mathrm{ml})$ of recombinant human SPAG9 (rhSPAG9) and evaluated for their phenotypic and functional ability. Subsequently, the efficacy of DCs primed with $750 \mathrm{ng} / \mathrm{ml}$ of rhSPAG9 (SPDCs) was compared with DCs primed with autologous tumour lysate (TLDCs), to induce CD4+, CD8 + T cells and activating NK cells. In addition, we investigated the effect of the chemotherapeutic drug cisplatin on phenotypic and functional potential of SPDCs.

\section{Results}

Phenotypic and functional characterization of DCs pulsed with $750 \mathrm{ng} / \mathrm{ml}$ rhSPAG9 was found to be optimal and effective for priming DCs. SPDCs were also capable of stimulating allogeneic CD $4+$ and CD8 $+T$ cells similar to TLDCs. SPDCs showed a statistically insignificant increase in the expression of maturation marker CD83 and migration towards CCL19 and CCL21 compared with TLDCs (CD83 $p=0.4$; for migration $p=0.2$ ). In contrast, TLDCs showed better proliferation and secretion of Th1 cytokines (IL12p40, IL12p70 and IFNY) compared to SPDCs, but this was also not statistically significant (IL12p40, $p=0.06)$. We also found that clinical dose of cisplatin $(200 \mu \mathrm{M})$ treated SPDCs were able to stimulate the proliferation of cytotoxic $\mathrm{T}$ lymphocytes without increasing the FOXP3 ${ }^{+}$Tregs in autologous co-cultures.

\section{Conclusions}

This is the first report to suggest that rhSPAG9 is an effective antigen for pulsing DCs that are capable of eliciting a potent Th1 response which, in turn, may help in decreasing the tumour burden when used along with a cisplatin based combinatorial regimen for therapeutic intervention. This strategy provides an insight to consider rhSPAG9 as a strong immunogen for DC-based immunotherapy for cervical cancer. 


\section{Background}

Cervical cancer is the leading cause of cancer related death among women worldwide and continues to be a major public health problem affecting middle-aged women, particularly in less-resourced countries [1]. Cervical cancer's cure rate is $90 \%$ in early stage disease. However, these rates drop to $50 \%$ or less in the advanced stages (IIlb and above) particularly in India [2]. Although a prophylactic vaccine is available, it is beyond the reach of most women in the developing countries due to financial implications. Novel therapeutic strategies that are effective in reducing the tumour burden are still needed desperately. Hence, we focused our efforts on developing a therapeutic vaccine for patients presenting with advanced stages of disease, which may be combined with existing concurrent chemo-radiotherapy regimens to improve cure rates.

Dendritic cells have emerged as important cell based therapeutic adjuvants due to their ability to cross present antigens to the host immune system and express ample co-stimulatory molecules. However, choice of tumour antigens has posed the biggest challenge to DC vaccine production. Hence the use of cancer testis antigen (CTA), due to their unique expression, have made them attractive peptide vaccine candidates, particularly MAGE-A and NY-ESO1 which have been used as therapeutic entities in several clinical trials [3-6]. Recent studies showed that restricted NY-ESO-1 immunogenic peptides in combination with various adjuvants exhibited potent anti-tumour response [7-8]. However, two other phase II trials with a single MAGEA3 peptide did not show an improvement in disease free survival [910]. In contrast to peptides or peptide mixtures, whole protein CT antigens may be suitable for peptide processing and presentation across several HLA types when used for priming autologous DCs [11-12].

Recently, sperm associated antigen 9 (SPAG9) has been shown to be expressed in various cancer types such as epithelial ovarian cancer (EOC- 90\%) [13], renal cell carcinoma (RCC- 88\%) [14], colorectal cancer (74\%) [15], breast cancer (88\%) [16] and cervical cancer (82\%) [17]. Studies on SPAG9 also showed that it is associated with cellular proliferation, migration and invasion of cancer cells [18-21], and is capable of eliciting humoral immune responses in a majority of epithelial ovarian cancers (67\%) [13], breast cancers (80\%) [16], cervical cancers (80\%) [17], renal cell carcinoma (77\%) [14], colorectal cancer (74\%) [15] and hepatocellular carcinoma [22]. However its antigenicity in invoking a cell mediated immune response has not been studied until now.

Combinatorial regimens utilizing chemotherapy and immunotherapy may decrease the tumour burden as well as the immunosuppressive cells in the tumour microenvironment facilitating pronounced synergistic effects. Several clinical trials conducted in solid tumours also showed an increased overall survival of patients when cell based immunotherapy with $D C$ vaccines was combined with chemotherapy regimens $[23,24]$. Hence evaluating the efficacy of dendritic cells and DC stimulated T cells in the presence of chemotherapeutic agents may be necessary to account for any difference in such functionality linked to specific antigens affecting their antigenicity.

This is the first study to assess the potential of SPAG9 protein as an antigenic source for dendritic cell priming. We primed immature DCs with increasing dosage of rhSPAG9 and determined the optimal 
priming dose. The efficacy of rhSPAG9 primed DCs (SPDC) was examined using immunophenotyping and functional assays. Our earlier studies in-vitro [25] and in phase I cervical cancer trials [26], we have demonstrated the efficacy of autologous tumour lysate pulsed DCs (TLDC). Hence, autologous TLDCs from the same patient were compared with SPDCs to assess their functional characteristics. In addition, the ability of SPDCs to elicit a Th1 response in combination with cisplatin at various concentrations was also assessed. Our present study has laid down the foundation for undertaking Phase II cervical cancer trials employing rhSPAG9 protein as an antigenic source for DC based vaccines.

\section{Materials And Methods}

\section{Patient samples}

Blood samples $(20 \mathrm{ml})$ were collected from 12 patients (Patient number P1-P12) who were diagnosed with cervical cancer at Cancer Institute Adyar, Chennai for isolation of PBMC and DC culture. Tumour punch biopsy samples were collected from 3 patients (Patient number P1-P3) to prepare tumour lysates for the generation of TLDCs. In addition, $20 \mathrm{ml}$ blood samples were also obtained from eight healthy donors for isolation of PBMCs using Ficoll (GE Healthcare, US). The monocytes were depleted by plastic adhesion subsequently to enrich for lymphocytes and used for allogeneic studies. The study was approved by the Institutional ethical committee from Cancer Institute Adyar, Chennai. The duly signed consent forms were obtained from each patient and healthy donor prior to the study. Tumour tissues were collected for tumour lysate in Hank's balanced salt solution (HBSS) containing $100 \mathrm{IU}$ of penicillin, streptomycin and gentamycin and in $10 \%$ formalin for immunohistochemistry.

\section{Immunohistochemistry for SPAG9 expression}

The SPAG9 protein expression was examined in cervical cancer tissue sections by $\mathrm{IHC}$ as described earlier [17]. Briefly, the cervical cancer tissue sections $(4 \mu \mathrm{m})$ were deparaffinized and subsequently rehydrated using different gradients of alcohol. Polyclonal antibodies to recombinant SPAG9 were generated and purified as described earlier [13]. Tissue sections were probed with anti-SPAG9 antibody or control IgG at $1: 100$ dilution overnight at $4^{\circ} \mathrm{C}$ in humid chamber. After washing thrice with phosphate buffer saline (PBS)-0.05\% Tween 20, the tissue sections were incubated with horseradish peroxidaseconjugated goat anti-rat IgG (Jackson ImmunoResearch Laboratories, West Grove, PA) and visualized using DAKO Envision kit (K500711-2) following the manufacturer's instructions and counterstained with hematoxylin and mounted with 1,3-diethyl-8-phenylxanthine [(DPX), Sigma-Aldrich, St. Louis, MO]. The images of tissue sections were captured using Nikon microscope (Nikon Instrument Inc., NY, USA).

\section{Antibodies and flow cytometry}

For phenotypic analysis of dendritic cells: Fluorochrome conjugated antibodies CD14-PC5 (CD-cluster of differentiation PC5- PE- Phycoerythrin cy5-cyanine, CD80-FITC, HLADR-ECD, CD40-PE and CD86-PE were purchased from Beckman Coulter Inc (CA, USA). The antibody against maturation marker CD83conjugated to APC was purchased from Bio Legend (San Diego, CA, USA) 
For phenotyping proliferating PBMCs, anti- CD56-PE, anti-CD4-PC5 anti-CD8-APC, anti- CD25-ECD and anti-CD3-PC7 antibodies were purchased from Beckman Coulter Inc. Anti-FOXP3-PE was purchased from BioLegend Inc. Flow cytometry was performed as described previously [25]. All the samples were acquired using MoFlo XDP flow cytometer configured with three different lasers - blue (488nm), violet $(405 \mathrm{~nm})$ red $(640 \mathrm{~nm})$ analyzed using Summit 5.2 software (both Beckman Coulter Inc).

\section{DC generation and priming}

Unprimed and TLDCs were generated from peripheral blood mononuclear cells (PBMCs) isolated using a Ficoll Paque Plus (GE Healthcare, UK) density gradient. Monocytes were enriched by plastic adherence for DC differentiation and were either primed with tumour lysates (TLDCs) prepared from single cell suspensions of punch biopsy samples or matured directly (unprimed DCs) as described earlier [25]. SPDCs were generated using GMP grade rhSPAG9 (GMP grade rhSPAG9 was outsourced for manufacturing to Syngene International Pvt. Ltd, India) [27]. The purified rhSPAG9 protein was used at different concentrations such as $250 \mathrm{ng}, 500 \mathrm{ng}, 750 \mathrm{ng}$ and $1000 \mathrm{ng} / \mathrm{ml}$ for priming and generation of SPDCs following same protocol used for the generation of TLDCs.

\section{Wash out test}

The mature dendritic cells were washed and suspended in serum free CellGro DC medium (Cell Genix, Freiburg, Germany) for $24 \mathrm{~h}$. Adherence and changes in morphology following cytokine withdrawal were monitored microscopically and counts were obtained by trypan blue exclusion. The washout test medium was stored in protein low bind tubes (Eppendorf, Hamburg, Germany) at $-70^{\circ} \mathrm{C}$.

\section{Phenotypic characterization of mature DCs}

For immunophenotypic analysis, $5 \times 10^{4}$ mature DCs were incubated for 10 min with $5 \%$ FBS and then stained with anti- CD14 PC5, CD40 PE, HLADR ECD, CD86 PE, CD80 FITC and anti CD83 APC for 20 min at RT. Cells were also stained with appropriate Isotypic controls and washed twice with PBS, fixed and suspended in $2 \%$ PFA (Sigma Aldrich, MI, USA) until analysis.

\section{Proliferation assay with rhSPAG9 primed DCs}

For the proliferation assay, non adherent cells enriched for lymphocytes after monocyte depletion for DC culture were prepared from allogenic and autologous PBMCs. Matured DCs primed with rhSPAG9 (250, 500, 750 and $1000 \mathrm{ng} / \mathrm{ml}$ per million cells), were cocultured with PBMCs stained with carboxy fluorescein, succinimidyl ester [CFSE (Invitrogen, CA, USA)]. Briefly, SPDCs were cocultured at the ratio of 1:50 (DC: allogeneic PBMCs) and 1:10 (DC: autologous PBMCs) to check for proliferation as described in our previous study [25].The cells were cultured for 8 days. The cells were centrifuged at $1580 \mathrm{rpm}$ and subsequently pellet was resuspended and washed with PBS, blocked with $5 \%$ FBS and stained with antiCD4-PC5, anti-CD8-APC, anti-CD56-PE, anti-CD25-ECD, anti-FOXP3-PE antibodies and propidium iodide $(1 \mu \mathrm{g} / \mathrm{ml}$; Himedia, India) to verify the response of the autologous or allogeneic PBMCs upon stimulation 
with SPDCs. Cells were acquired using a MoFlo XDP cell sorter/ Flow cytometer (Beckman Coulter) and FCS express 7 was used for analyzing the proliferating population.

\section{Migration assay}

Migratory capacity of TLDCs and SPDCs was assessed in a 24 well plate using $(5 \mu)$ transwell inserts (Corning, NY, USA). The lower chamber of each well was filled with serum free medium containing $300 \mathrm{ng} / \mathrm{ml} \mathrm{CCL} 19$ and 250ng/ml CCL21 (R\&D systems, MN, USA) and the DCs were incubated for $3 \mathrm{~h}$ at $37^{\circ} \mathrm{C}$. Subsequently, the cells were centrifuged and then re-suspended in total volume of $300 \mu \mathrm{l}$ PBS. Samples were analyzed in a flow cytometer using a fixed flow rate. The number of cells acquired per minute through flow cytometer was calculated as described previously [25].

\section{IFNY Analysis by ELISPOT assay}

ELISPOT assay was done to measure antigen-specific IFNy release by PBMCs removed after monocytes attachment. All steps were carried out as per manufacturer's protocol [CTL Technologies, OH, USA]. The matured DCs (TLDCs or SPDCs or unprimed DCs) were incubated with allogenic non adherent PBMCs at a 1:50 ratio for $24 \mathrm{~h}$ at $37^{\circ} \mathrm{C} / 5 \% \mathrm{CO}_{2}$ in IFNy captured antibody coated wells. The lymphocyte enriched autologous PBMCs were also cultured similarly with matured DCs at 1:10 ratio for 2 weeks and incubated for $48 \mathrm{~h}$ in IFNy captured antibody coated wells in the presence of $100 \mathrm{IU} / \mathrm{ml} \mathrm{IL2}$ along with respective matured DCs. To avoid inter sample variation we multiplied the spot count with the spot size which is represented as the IFNy spot Index for each patient. Following manufacturer's instructions spots were scanned using Immunospot versa analyser (CTL, OH, USA) using ImmunoCapture software version 6.4. The spots were then counted using the ImmunoSpot 5.0 ProDC software.

\section{Cytokines Analysis by ELISA}

Patients (patient number P7-P9) monocytes derived DC either primed with rhSPAG9 or tumour lysates were generated in 7 days. Post 7 days, SPDCs and TLDCs were cultured in plain Cell Gro medium for $24 \mathrm{~h}$ without the proinflammatory cytokine. All viable floating matured DCs were collected for DCs-PBMCs cocultures experiments and supernatant (WO) was stored at $-80^{\circ} \mathrm{C}$ for conducting the ELISA experiments. Subsequently, autologous DCs thus generated were further co-cultured with PBMCs for 7 days. Supernatants from each well were collected on Day 7 and stored at $-80^{\circ} \mathrm{C}$ for conducting ELISA experiments. Both IL12p70 and p40 were quantitated separately using the respective ELISA kits (IL12p70 and IL12p40 kit; Biolegend, CA, USA) according to the manufacturer's instructions. The color developed was read at 450nm using Multiskan Ascent (Thermo Electron Corporation, USA).

\section{Effect of Cisplatin treatment on autologous PBMC cocultures with dendritic cells}

To examine the effect of Cisplatin (Kemoplat, Fresenius Kabi, Germany) on DC function, three different

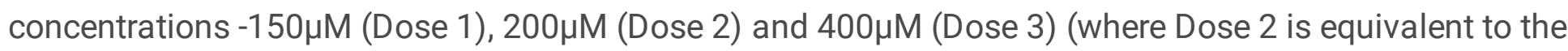
clinical dosage) were used. SPDCs were seeded ( $1 \times 10^{5}$ cells) into 96 well plate and cultured for $24 \mathrm{~h}$ at 
$37^{0} \mathrm{C}$ followed by three different doses of cisplatin treatment. Treated SPDC were cocultured with autologous PBMC in 1:10 ratio ( $n=3$; patient number P10-12). The frequency of treatment was day 0,3 and 5 for monitoring CFSE based proliferation, for up to eight generations which is usually attained within a week. DCs alone as control were treated for $48 \mathrm{~h}$ with cisplatin and phenotypic marker expression and migratory capacity were analyzed. All experiments were carried out in three independent patients (P10-P12).

\section{Statistical analysis}

Mann-Whitney $U$ test was done to compare unprimed DCs with SPDCs and TLDC with SPDCs. One-way ANOVA (Analysis of variance) was used to compare the phenotypic and functional characteristics of untreated and cultures treated with three different doses of CDDP. All the analyses were done using GraphPad prism 7 software (GraphPad Software, Inc. USA).

\section{Results}

\section{SPAG9 protein expression in cervical cancer tissue}

SPAG9 protein expression was probed in 12 cervical cancer patients using IHC. For all further in vitro studies, tumour lysates, DCs and PBMC were also obtained from these patients. Our histopathological analysis showed that two patients were found to have poorly differentiated carcinoma with SPAG9 protein expression. The other 10 patients diagnosed with squamous cell carcinoma grade II/III (Table 1) also expressed SPAG9 but with varying intensity. SPAG9 immunoreactivity staining was graded on the following scale: 0 , no staining; $1+$, weak staining; $2+$, moderate staining; $3+$, intense staining (Figure 1 ). Our results indicated that SPAG9 protein was expressed in all the patients irrespective of histotypes.

\section{Phenotypic characterization of SPAG9 primed matured DCs}

Initially, we evaluated the phenotypic characterization of rhSPAG9 primed DCs at four different concentrations $(250,500,750$ and $1000 \mathrm{ng} / \mathrm{ml})$. The percentage of live DCs primed with rhSPAG9 post 72 hours was calculated. The highest yield of live DCs in culture media was observed with $750 \mathrm{ng} / \mathrm{ml}$ of rhSPAG9 as compared to other concentration of rhSPAG9 (Figure 2a).

As previously known, the expression of CD14 was completely down regulated in mature DCs irrespective of antigen priming [25]. There was no difference in the expression of the evaluated phenotypic markers between SPAG9 primed and unprimed DCs. However, when we performed an analysis within DCs primed with different concentrations of rhSPAG9, we found that, CD80 expression alone was slightly albeit insignificantly increased when 750ng and $1000 \mathrm{ng} / \mathrm{ml}$ of rhSPAG9 was used for DC priming (Figure 2b).

\section{Optimization of rhSPAG9 concentration for DCs priming based on proliferation of allogeneic PBMCs}

DCs were primed with various concentrations of rhSPAG9 $(250,500,750$ and $1000 \mathrm{ng} / \mathrm{ml})$ that revealed DCs primed with rhSPAG9 at a concentration of $500 \mathrm{ng}$ and $750 \mathrm{ng} / \mathrm{ml}$ were having similar ability to 
induce proliferation of allogeneic PBMCs compared to those primed with 250 and, $1000 \mathrm{ng} / \mathrm{ml}$. Further, we analyzed the status of $\mathrm{CD} 4^{+}$and $\mathrm{CD} 8^{+} \mathrm{T}$ cells in the dividing population. We observed that the $\mathrm{CD} 8^{+} \mathrm{T}$ cell response was slightly higher in cells stimulated with 500ng of rhSPAG9, whereas both CD $4^{+}$and $\mathrm{CD}^{+} \mathrm{T}$ cells response was higher in DCs primed with 750ng of rhSPAG9 (Figure 2c). Hence, we selected $750 \mathrm{ng}$ concentration of rhSPAG9 protein to prime DCs in vitro.

Subsequently, in order to ascertain the phenotypic characteristics of SPDCs with reference to autologous TLDC, we compared the SPDCs primed with $750 \mathrm{ng} / \mathrm{ml}$ rhSPAG9 with TLDCs in vitro. We did not find the difference in expression of CD80, CD86, CD40 and HLA-DR but CD83 alone had an insignificant increase in SPDCs compared to TLDCs ( $p$ value $=0.4)$ (Figure $2 d)$.

We further examined the functional efficacy. Our data revealed no significant difference in proliferation of allogeneic PBMCs stimulated with either TLDCs or SPDCs (Figure 2e). The number of $C D 4^{+} \mathrm{T}$ and $\mathrm{CD} 8+\mathrm{T}$ cells was also similar in allogeneic PBMCs, stimulated with either TLDCs or SPDCs (Figure 2f).

\section{Secretion of IFNY by allogenic PBMCs}

SPDCs and TLDCs from patients (P7-P9) were prepared and co-cultured with allogenic PBMCs to quantitate IFNY-secretion. SPDCs and TLDCs prepared from patient No 7 were co-cultured with allogenic PBMCs isolated from normal healthy donor (N1). Similarly, SPDCs and TLDCs prepared from patient No. 8 and 9 were co-cultured with allogenic PBMCs isolated from normal healthy donors N2 and N3, respectively. Our data revealed that the spot index of IFNY was similar in TLDCs and SPDCs derived from patients P8 and P9 and co-cultured with allogenic N2 and N3, (Figure 2g). However, with allogenic PBMC from N1, we observed that TLDC induced cultures had a four-fold difference compared to SPDC induced cultures.

\section{Migratory response of mature DC toward CCL19 and CCL21}

The experiments were performed in three independent patient samples (P7-P9). Interestingly, our results showed the migratory response of SPDCs to be insignificantly higher ( $70 \pm 12 \% ; n=3$; P7-P9) when compared to TLDCs $(57 \pm 19.5 \%$; $=3$; P7-P9) $(p=0.2)$, indicating that SPDCs and TLDCs were capable of up regulating CCR7 in response to the chemokine cocktail CCL19 and CCL21 (Figure 3a-b).

\section{Functional characterization of SPDCs and TLDCs: proliferation of autologous PBMCs}

Autologous PBMCs proliferation response was examined with cocultures of SPDCs and TLDCs. The mean percentage of overall proliferation in TLDCs was $13.56( \pm 10.8)$ whereas it was $10.34 \%( \pm 6.4)$ in SPDC co-cultures as depicted in figure $4 a(n=3 ; P 7-P 9)$. We found a similar NK cells $\left(C D 56^{+}\right)$response in both cocultures; $C D 8^{+} T$ and $C D 4+T$-cell proliferation were similar in two patients (P8 and P9). In one patient (P7) CD8+T cells showed higher proliferation upon SPDCs stimulation whereas in the same patient (P7), CD4+T cell proliferation was increased when stimulated with TLDCs (Figure 4b). 


\section{IFNy, IL12p40 and IL12p70 secretory response of autologous PBMCs}

For three patients, we evaluated the cytokine profile of TLDCs and SPDCs in cytokine withdrawn wash out (WO) supernatants after $24 \mathrm{~h}$ and day 7 cocultures using ELISA. We observed that the secretion of IL12p40 was higher in the SPDCs- WO supernatants compared to the same from TLDCs (Figure $4 \mathrm{c}$ ). However, the co-culture supernatants of TLDCs at day 7 showed higher levels of IL12p40 secretion when compared with SPDCs. In contrast, the secretion of IL12p70 was minimal in all the WO supernatants of TLDCs and SPDCs. (Figure 4d). When we compared the ratio of IL12p40/p70 of SPDCs and TLDCs co cultures, it was 19.5 and 21.5 (P8) and 8.5 and 7.8 (P9) indicating that the response to both TLDCs and SPDCs was similar. However, in the third patient (P7), IL12p40 /p70 secretion was higher in TLDCs (595.7) compared to the SPDCs. When we cross checked these results with IFN gamma secretion, patient (P7) also had the highest spot Index in TLDCs compared to SPDCs (figure 4e). We observed that CD4+ cells had proliferated in greater numbers ( $>4$ fold) in TLDCs co-cultures of the same patient when compared to those stimulated by SPDCs (Figure $4 b$ ).

Immuno-histochemical analysis showed that all the three patients (P7, 8 and 9) had similar levels of SPAG9 expression in their tumours (80-90\%) with $2+$ intensity (Table 1 ), leading us to speculate that tumour lysates could have a varying mixture of tumour associated antigens with differing antigenicity leading to variation in response levels compared to the single protein antigen rhSPAG9

\section{Evaluating the cytotoxic effect of cisplatin on SPDCs}

We further evaluated the effect of cisplatin on SPDCs phenotype, viability, as well as their migratory capacity. Our results indicated that Dose $2(200 \mu \mathrm{M})$ and Dose $3(400 \mu \mathrm{M})$ cisplatin treated SPDCs showed an increase in cell death compared to the untreated controls. However, this was not significant. Further, the costimulatory or maturation markers as well as their migratory capacity was not significantly affected in any of the cisplatin treated SPDCs. We however found an insignificant decrease in CD80 and increase in HLADR expression upon cisplatin treatment. This showed that 48 hours after cisplatin treatment, the SPDCs retained their viability, phenotypic and migratory characteristics (Figure $5 \mathrm{a}, \mathrm{b}$ ).

In addition to phenotypic expression we also analyzed the proliferation of autologous PBMCs upon SPDCs stimulation in the presence or absence of cisplatin. The overall proliferation of Dose 2 and Dose 3 cisplatin treated SPDCs culture was decreased compared to the Dose $1(150 \mu \mathrm{M})$ and untreated cultures. However, the decrease was not significant among the cisplatin treated cultures compared to untreated cocultures (Figure $5 \mathrm{c}, \mathrm{d}$ ). When we analyzed the T and NK cell $\left(\mathrm{CD} 56^{+}\right)$compartments, we observed a decrease in the highest dose $(400 \mu \mathrm{M})$ of cisplatin treated cultures alone ( $P$ value $=0.02)$ (Figure $5 \mathrm{e}$ ). Interestingly, we observed an increased proliferation of $\mathrm{CD} 4^{+}$and $\mathrm{CD} 8^{+} \mathrm{T}$ cells in Dose 2 treated cocultures (Figure 5e). We further checked if an increase in the $\mathrm{FOXP3}^{+}$compartment led to the rise in proliferation. We calculated the ratio of total $\mathrm{CD} 3+/ \mathrm{FOXP3}+\mathrm{T}$ cell population and found a slight decrease in the ratio only in Dose 3 treated co cultures. However, we speculate the decrease in the ratio could be due to an overall increase in the death of proliferating $T$ cells at the highest dosage (Figure $6 a, b$ ) 
indicating that Tregs did not undergo proliferation and hence were not affected by the highest dose of cisplatin.

\section{Discussion}

Cervical cancer is one of the major gynecological cancers leading to cancer related deaths worldwide [1]. Majority of cervical cancers are diagnosed at advanced stage with poor prognosis and high morbidity [1]. Recently, SPAG9 was shown to be associated with cervical cancer ${ }^{17}$ which also showed that majority of the patient's generated humoral response against SPAG9 protein suggesting that SPAG9 is a highly immunogenic protein. These findings are thus suggestive of SPAG9 as potential target for the development of novel therapeutic interventions. In the current study, our results confirmed the SPAG9 protein expression in all histotypes and grades of cervical cancer patients who were included for this investigation. The goals of cancer immunotherapy are to activate and expand tumour-specific $\mathrm{CD} 4^{+}$and $\mathrm{CD}^{+} \mathrm{T}$ cells as effective means of augmenting immunity to reduce tumour burden. Therefore, initially we examined the various concentrations of rhSPAG9 for generating SPDCs that showed effective Th1 polarization with $750 \mathrm{ng} / \mathrm{ml}$ of rhSPAG9 in the presence of proinflammatory cytokines. In addition, the generated SPDCs also possessed comparable expression of both mature and costimulatory markers with increased migratory capacity. We also observed that the SPDCs were capable of inducing a Th1 response both in allogenic and autologous responders by increasing the proliferation of CD8 + CTL compared to T helper cells. We also confirmed that the cisplatin treatment at $150 \mu \mathrm{M}$ and $200 \mu \mathrm{M}$ concentration, which is used as the chemotherapeutic agent in cervical cancer treatment, did not inhibit the efficacy of SPDCs. In addition, cisplatin at a concentration of $150 \mu \mathrm{M}$ and $200 \mu \mathrm{M}$ treatment did not impede significantly the proliferation of $\mathrm{CD} 4^{+}$and $\mathrm{CD} 8^{+} \mathrm{T}$ cells.

Priming of DC with whole protein or whole tumour lysates from autologous tumours have been used in treating numerous cancers. The major advantages of this method compared to peptides are the multiple epitopes presented by $\mathrm{MHC}$ molecules that can activate both $\mathrm{CD} 4^{+}$and $\mathrm{CD} 8^{+} \mathrm{T}$ cell effectively towards a wide spectrum of antigens. Along with this, the requirement for processing of antigens results in prolonged antigen presentation [28]. The use of whole cancer-testis antigen proteins such as NY-ESO-1 and MAGEA3 when given alone were found mediating both humoral and cellular immune response [29]. A recent phase I study conducted in advanced cancers showed that administration of LV305, a novel lentivirus based cancer vaccine that induces the expression of NY-ESO1 in DCs, resulted in potential clinical activity with the induction of antigen specific response in sarcoma, melanoma, ovarian and lung cancers [34]. Our current study shows that the priming of DCs with the novel rhSPAG9, showed comparable increase in maturation markers with statistically insignificant increased migratory potential in comparison to whole tumour lysate pulsed DCs. Some maturation cocktails contain PGE2 in order to improve migratory properties of DCs. We found SPDCs to be surprisingly more robust at migrating towards the CCR7 ligands CCL19 and 21, across a chemokine gradient even without PGE2 in the maturation cocktail. PGE2 is controversial for its ability to cause infiltration of suppressive cells 
expressing the enzyme Indoleamine 2,3, dioxygenase (ID01) which in turn caused the infiltration of FOXP3 + regulatory cells [31].

SPDCs were capable of eliciting a potent Th1 response even at a concentration of $750 \mathrm{ng} / \mathrm{ml}$. However, in comparison, studies have shown that peptides need to be used at a much higher concentration (up to $10 \mathrm{ug} / \mathrm{ml}$ ) [32]. Several studies also reported that CTAs have restriction epitopes identified as the recognition sites for $C D 8^{+} T$ lymphocytes [33-35]. Similarly, our in-silico analysis also showed the presence of CD8 epitopes in SPAG9 protein (data not published) which was supported by higher CD8 proliferation and was accompanied by IFNy secretion in vitro.

A clinical trial conducted in pancreatic cancer patients treated with a combinatorial treatment using the Onivyde and leucovorin /5FU regimen followed by 6 doses of autologous dendritic cell vaccine showing a substantial decrease in the tumour burden [36]. In addition, studies also showed a reduction in Treg/MDSC cells along with tumour specific CD $4+$ and $C D 8+T$ cell generation in patients when dendritic cell vaccines were combined with chemotherapeutic regimen [37-39]. In this context we studied the effect of cisplatin on SPAG9 primed DCs and the responding immune cells. Our study showed that cisplatin treatment did not downregulate phenotypic expression even at higher concentration. Further, the percentage of HLADR was slightly increased in cisplatin treated cultures compared to untreated cells which are in line with the results reported earlier [40], that showed an increase in the expression of HLA class II upon treatment of dendritic cells with paclitaxel [38]. In a different study, it was reported that addition of cisplatin during DC differentiation did not inhibit T cell proliferation [41]. Our study also shows that cisplatin treatment of the differentiated rhSPAG9 primed DCs did not inhibit their ability to stimulate helper and cytotoxic cells to proliferate.

Similarly, several studies [42-43] have shown the importance of ratio between CD4+/, CD8+/ or CD3+/ and FOXP3 + cells as being crucial in predicting prognosis of cancer patients. Hence, we wanted to verify the effect of SPDC stimulation in-vitro on the CD3+/FOXP3 + ratio in the presence or absence of cisplatin. However we did not find any significant decrease in the ratio at the clinical dose equivalent. This presents the possibility of combining vaccination using SPDCs with a cisplatin based chemotherapeutic regimen in the treatment of advanced cervical cancer.

In conclusion, this preclinical report provides evidence of a potent Th1 response in vitro, produced by the novel rhSPAG9 protein primed DC similar to that of TLDC, and may be combined with the chemotherapeutic drug cisplatin for the treatment of cervical cancer patients in advanced stages of the disease.

\section{Abbreviations}

ANOVA-Analysis of variance

APC- Allophycocyanin 
CD- Cluster of differentiation

CDDP- Cis-di amine di chloride platinum (II)

DCs - Dendritic cells

ECD- Energy Coupled Dye

ELISA- Enzyme-Linked Immunosorbent assay

ELISpot- Enzyme-Linked Immune absorbent spot

FITC - Fluorescein isothiocyanate

FOXP3- Fork head box P3

GMCSF- Granulocyte-Macrophage colony stimulation factor

HPV-Human papilloma virus

IFN- Interferon

IL- Interleukin

MHC- Major Histocompatibility complex

MIP- Macrophage inflammatory protein

NK-Natural killer

PBMCs- Peripheral blood mononuclear cells

PC5- Phycoerythrin Cyanine 5

PC7- Phycoerythrin Cyanine 7

PE- Phycoerythrin

SPDC-SPAG9 primed DCs

TLDCs-Tumour Lysate Dendritic Cells

TLR- Toll like receptor

TNF- Tumour necrosis factor

Tregs- T regulatory cells 
TS- Type specific

WIA- Women's India Association

WO- Wash out

\section{Declarations}

\section{Ethics approval and consent to participate}

The study was approved by the Institutional ethical committee from Cancer Institute Adyar, Chennai. The duly signed consent forms were obtained from each patient and healthy donor prior to the study.

\section{Consent for publication}

Not applicable

\section{Availability of data and materials}

Not applicable

\section{Competing interests}

The authors declare that they have no competing interests.

\section{Funding}

The author(s) disclosed receipt of the following financial support for the research, authorship, and/or publication of this article: This work was supported by grant from Department of Science and Technology, Government of India (Grant no: SR/S9/Z-08/2010 dated 25-06-2010) to Dr T Rajkumar, Cancer Institute (WIA). Dr Anil Suri, NII was supported by the Indo-UK Cancer Research Program (Grant No. BT/IN/UK/NII/2006) and NII-core funding, Department of Biotechnology, Government of India. Ms. Hemavathi was awarded fellowship from Council of Science and Industrial Research. Ms. Abirami was awarded fellowship from Department of Science and Technology under the Inspire Fellowship scheme.

\section{Author contributions}

H.D, H.J and A.Se carried out all the experiments, prepared figures and drafted the manuscript. G. S evaluated and recruited patients for the study, provided samples and critically reviewed the manuscript. N.J, and A.Su collaborated for rhSPAG9 synthesis, and critically reviewed the manuscript. S.S.S did the histopathological and Immunohistochemical assessment. T.R and P.R designed the study, involved in data analysis, preparation and review of manuscript.

\section{Acknowledgements:}


We acknowledge Dr. Vasanth Christopher, Cancer Institute, Chennai, for his support during patient recruitment and sample collection.

\section{Conflict of Interest}

The authors declare that they have no conflict of interest.

\section{References}

1. Arbyn M, Weiderpass E, Bruni L, Sanjosé S, Saraiya M, Ferlay J, Bray F. Estimates of incidence and mortality of cervical cancer in 2018: a worldwide analysis. Lancet Glob Health. 2020;8:e191-203.

2. Ramanathan $P$, Dhandapani $H$, Jayakumar $H$, Seetharaman $A$, Thangarajan R. Immunotherapy for cervical cancer: Can it do another lung cancer? Curr Probl Cancer. 2018;42(2):148-60.

3. Jäger E, Karbach J, Gnjatic S, et al. Recombinant vaccinia / fowlpox NY-ESO-1 vaccines induce both humoral and cellular NY-ESO-1-specific immune responses in cancer patients. Proc Natl Acad Sci USA. 2006;103(39):14453-8.

4. Odunsi K, Matsuzaki J, Karbach J, et al. Efficacy of vaccination with recombinant vaccinia and fowlpox vectors expressing NY-ESO-1 antigen in ovarian cancer and melanoma patients. Proc Natl Acad Sci USA. 2012;109(15):5797-802.

5. Study of MAGE-A3. and NY-ESO-1 immunotherapy in combo with DTPACE chemo and auto transplantation in multiple myeloma - Full Text View - ClinicalTrials.gov. https://clinicaltrials.gov/ct2/show/NCT00090493.

6. McQuade JL, Homsi J, Torres-Cabala CA, et al. A phase II trial of recombinant MAGE-A3 protein with immunostimulant AS15 in combination with high-dose Interleukin-2 (HDIL2) induction therapy in metastatic melanoma. BMC Cancer. 2018;18(1):1274.

7. Sonpavde G, Wang M, Peterson LE, et al. HLA-restricted NY-ESO-1 peptide immunotherapy for metastatic castration resistant prostate cancer. Invest New Drugs. 2014;32(2):235-42.

8. Bender A, Karbach J, Neumann A, et al. LUD 00-009: phase 1 study of intensive course immunization with NY-ESO-1 peptides in HLA-A2 positive patients with NY-ESO-1-expressing cancer. Cancer Immun. 2007;7:16.

9. Tyagi P, Mirakhur B. MAGRIT: the largest-ever phase III lung cancer trial aims to establish a novel tumor-specific approach to therapy. Clin Lung Cancer. 2009;10(5):371-4.

10. Dreno B, Thompson JF, Smithers BM, et al. MAGE-A3 immunotherapeutic as adjuvant therapy for patients with resected, MAGE-A3-positive, stage III melanoma (DERMA): a double-blind, randomised, placebo-controlled, phase 3 trial. Lancet Oncol. 2018;19(7):916-29.

11. Laidlaw BJ, Craft JE, Kaech SM. The multifaceted role of CD4 (+) T cells in CD8 (+) T cell memory. Nat Rev Immunol. 2016;16(2):102-11.

12. León B, Ballesteros-Tato A, Randall TD, Lund FE. Prolonged antigen presentation by immune complex-binding dendritic cells programs the proliferative capacity of memory CD8 T cells. J Exp 
Med. 2014;211(8):1637-55.

13. Garg M, Chaurasiya D, Rana R, et al. Sperm-associated antigen 9, a novel cancer testis antigen, is a potential target for immunotherapy in epithelial ovarian cancer. Clin Cancer Res. 2007;13(5):1421-8.

14. Garg M, Kanojia D, Khosla A, et al. Sperm-associated antigen 9 is associated with tumor growth, migration, and invasion in renal cell carcinoma. Cancer Res. 2008;68(20):8240-8.

15. Kanojia D, Garg M, Gupta S, Gupta A, Suri A. Sperm-Associated Antigen 9 is a novel biomarker for colorectal cancer and is involved in tumor growth and tumorigenicity. The Am $\mathrm{J}$ of Pathol. 2011;178(3):1009-20.

16. Kanojia D, Garg M, Gupta S, Gupta A, Suri A. Sperm-associated antigen 9, a novel biomarker for early detection of breast cancer. Cancer Epidemiol Biomarkers Prev. 2009;18:630-9.

17. Garg M, Kanojia D, Salhan S, Suri S, Gupta A, Lohiya NK, Suri A. Sperm-associated antigen 9 is a biomarker for early cervical carcinoma. Cancer. 2009;115:2671-83.

18. Suri A, Saini S, Sinha A, et al. Cancer testis antigens: A new paradigm for cancer therapy. Oncoimmunology. 2012;1(7):1194-6.

19. Sinha A, Agarwal S, Parashar D, et al. Down regulation of SPAG9 reduces growth and invasive potential of triple-negative breast cancer cells: possible implications in targeted therapy. J Exp Clin Cancer Res. 2013;32(1):69.

20. Jagadish N, Fatima R, Sharma A, Devi S, Suri V, Kumar V, Suri A. Sperm associated antigen 9 (SPAG9) a promising therapeutic target of ovarian carcinoma. Tumour Biol. 2018;40(5):1010428318773652.

21. Jagadish N, Gupta N, Agarwal S, Parashar D, et al. Sperm-associated antigen 9 (SPAG9) promotes the survival and tumor growth of triple-negative breast cancer cells. Tumour Biol. 2016;37(10):13101-10.

22. Ren B, Zou G, Xu F, et al. Serum levels of anti-sperm-associated antigen 9 antibody are elevated in patients with hepatocellular carcinoma. Oncol Lett. 2017;14(6):7608-14.

23. Qiao G, Wang X, Zhou L, et al. Autologous Dendritic Cell-Cytokine Induced Killer Cell Immunotherapy Combined with S-1 Plus Cisplatin in Patients with Advanced Gastric Cancer: A Prospective Study. Clin Cancer Res. 2019;25(5):1494-504.

24. Zhou X, Mo X, Qiu J, et al. Chemotherapy combined with dendritic cell vaccine and cytokine-induced killer cells in the treatment of colorectal carcinoma: a meta-analysis. Cancer Manag Res. 2018;10:5363-72.

25. Ramanathan P, Dhandapani H, Jayakumar H, Ganeshrajah S, Thangarajan R. Dendritic cells primed with HPV positive cervical tumor lysate are superior to unprimed DCs in migratory capacity and induce a potent Th1 response. Hum Immunol. 2014;75(12):1216-24.

26. Ramanathan P, Ganeshrajah S, Raghanvan RK, Singh SS, Thangarajan R. Development and clinical evaluation of dendritic cell vaccines for HPV related cervical cancer-a feasibility study. Asian Pacific Journal of Cancer Prevention: APJCP. 2014;15(14):5909-16. 
27. Jagadish N, Rana R, Selvi R, Mishra D, Garg M, Yadav S, Herr JC, Okumura K, Hasegawa A, Koyama K. Suri A.Characterization of a novel human sperm-associated antigen 9 (SPAG9) having structural homology with c-Jun N-terminal kinase-interacting protein. Biochem J. 2005;389(1):73-82.

28. Osada T, Clay TM, Woo CY, Morse MA, Lyerly HK. Dendritic cell-based immunotherapy. Int Rev Immunol. 2006;25(5-6):377-413.

29. Wei X, Chen F, Xin K, et al. Cancer-Testis Antigen Peptide Vaccine for Cancer Immunotherapy: Progress and Prospects. Transl Oncol. 2019;12(5):733-8.

30. Somaiah N, Block MS, Kim JW, et al. First-in-Class, First-in-Human Study Evaluating LV305, a Dendritic-Cell Tropic Lentiviral Vector, in Sarcoma and Other Solid Tumors Expressing NY-ESO-1. Clin Cancer Res. 2019;25(19):5808-17.

31. Braun D, Longman RS, Albert ML. A two-step induction of indoleamine 2, 3 dioxygenase (IDO) activity during dendritic-cell maturation. Blood. 2005;106(7):2375-81.

32. Mirandola L, Chiriva-Internati M, Bresalier R, et al. Preliminary report of a novel formulation of clinical-grade, fully matured, tumor-associated peptide-loaded dendritic cells for cancer immunotherapy. Transl Med Commun. 2019;4:17.

33. Yamaguchi $H$, Tanaka F, Ohta M, Inoue H, Mori M. Identification of HLA-A24-restricted CTL epitope from cancer-testis antigen, NY-ESO-1, and induction of a specific antitumorimmune response. Clin Cancer Res. 2004;10(3):890-6.

34. Eikawa S, Kakimi K, Isobe M, et al. Induction of CD8 T-cell responses restricted to multiple HLA class I alleles in a cancer patient by immunization with 20-mer NY-ESO-1f (NY-ESO-1 91-110) peptide. Int J Cancer. 2013;132(2):345-54. doi:10.1002/ijc.27682.

35. Sadanaga N, Nagashima H, Mashino K, et al. Dendritic cell vaccination with MAGE peptide is a novel therapeutic approach for gastrointestinal carcinomas. Clin Cancer Res. 2001;7(8):2277-84.

36. Chiriva-Internati M, Mirandola L, Bresalier R, et al. Combination therapy of dendritic cell vaccination plus chemotherapy in pancreatic cancer. J Clin Oncol. 2019;37:e15748-8.

37. Matsushita $\mathrm{H}$, Enomoto $\mathrm{Y}, \mathrm{Kume} \mathrm{H}$, et al A pilot study of autologous tumor lysate-loaded dendritic cell vaccination combined with sunitinib for metastatic renal cell carcinoma. J Immunother Cancer. 2014; 2:30. Yanagisawa R, Koizumi T, Koya T, et al. WT1-pulsed Dendritic Cell Vaccine Combined with Chemotherapy for Resected Pancreatic Cancer in a Phase I Study. Anticancer Res. 2018; 38(4):22172225.

38. Zhang L, Xu Y, Shen J, et al. Feasibility study of DCs/CIKs combined with thoracic radiotherapy for patients with locally advanced or metastatic non-small-cell lung cancer. Radiat Oncol. 2016;11:60.

39. John J, Ismail M, Riley C, et al. Differential effects of Paclitaxel on dendritic cell function. BMC Immunol. 2010;11:14.

40. Hu J, Kinn J, Zirakzadeh AA, et al. The effects of chemotherapeutic drugs on human monocytederived dendritic cell differentiation and antigen presentation. Clin Exp Immunol. 2013;172(3):490-9.

41. Sinicrope FA, Rego RL, Ansell SM, Knutson KL, Foster NR, Sargent DJ. Intraepithelial effector $(\mathrm{CD} 3+)$ /regulatory (FoxP3+) T-cell ratio predicts a clinical outcome of human coloncarcinoma. 
Gastroenterology. 2009;137(4):1270-9.

42. Sideras K, Galjart B, Vasaturo A, et al. Prognostic value of intra-tumoral CD8+ /FoxP3 + lymphocyte ratio in patients with resected colorectal cancer liver metastasis. J Surg Oncol. 2018;118(1):68-76.

43. Chen WY, Wu CT, Wang CW, et al. Prognostic significance of tumor-infiltrating lymphocytes in patients with operable tongue cancer. Radiat Oncol. 2018;13(1):157.

\section{Figures}

\section{Figure 1}
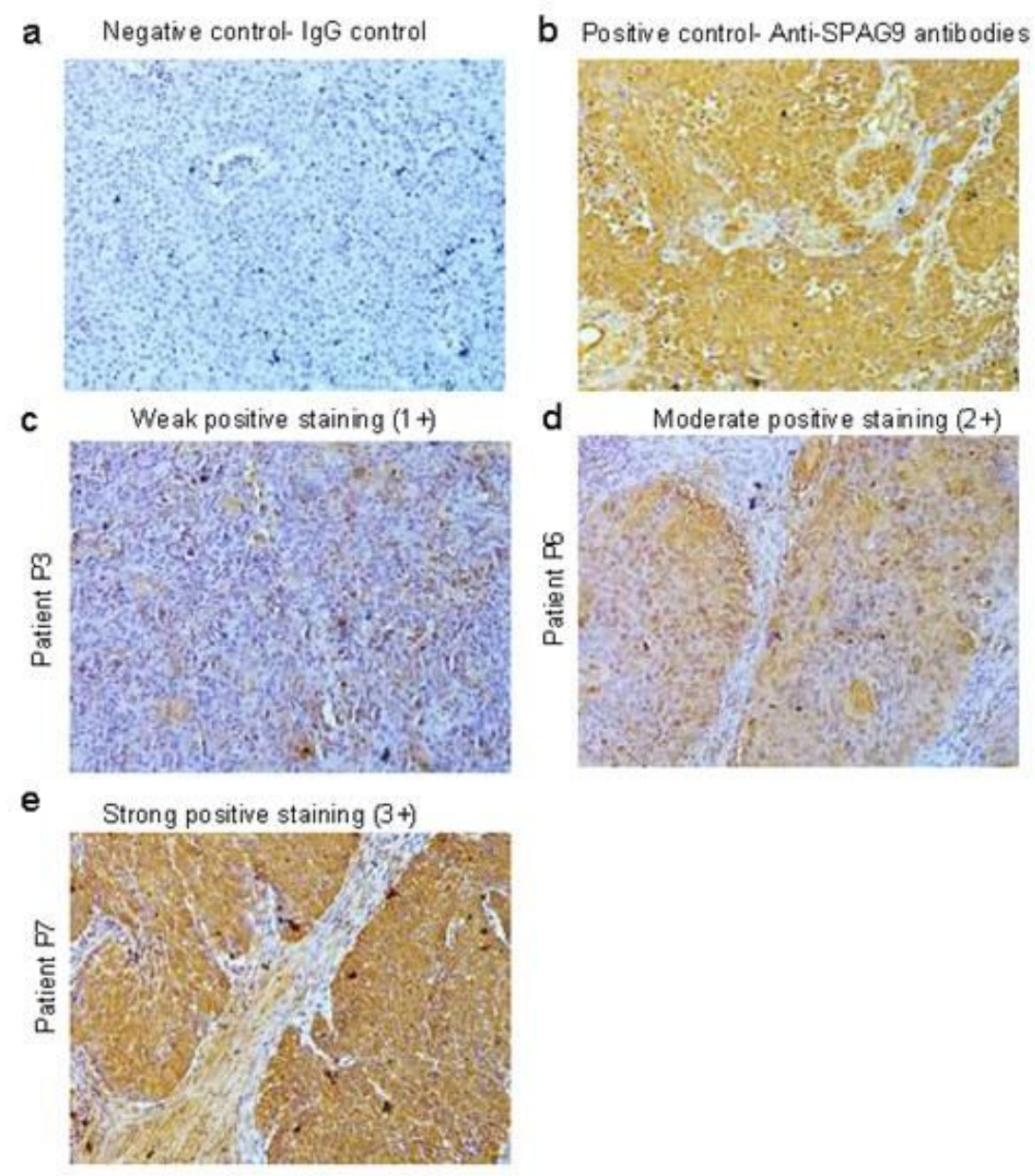

\section{Figure 1}

Immunohistochemical analysis of SPAG9 expression in cervical cancer tissues (a) Negative control (stained with secondary antibody only) (b) representative image of positive control stained with antiSPAG9 antibody as positive control (c) representative image of weak positive staining intensity (1+) probed with anti-SPAG9 antibody (d) representative image of moderate positive staining intensity (2+) 
probed with anti-SPAG9 antibody (e) representative image of strong positive staining with intensity (3+) probed with anti-SPAG9 antibody.

Figure 2

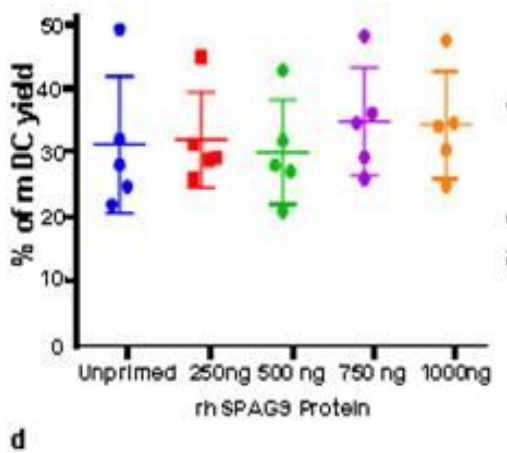

d

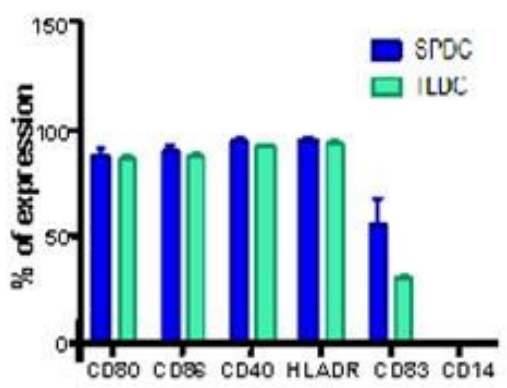

9

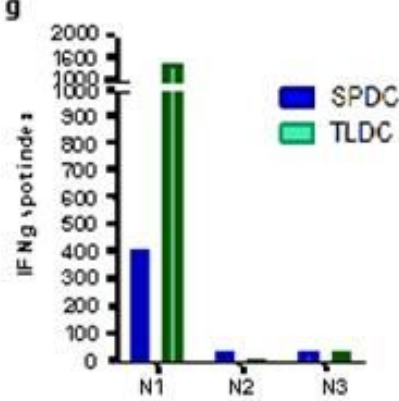

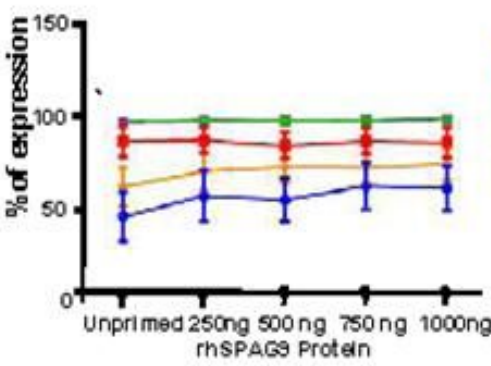

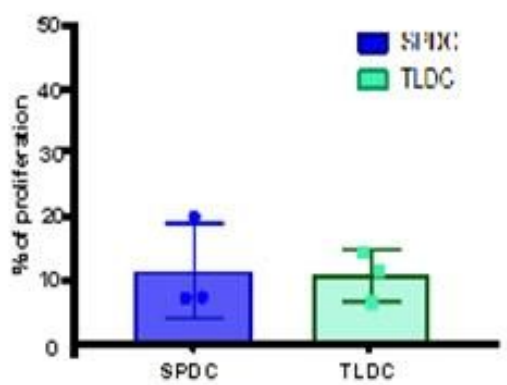

c
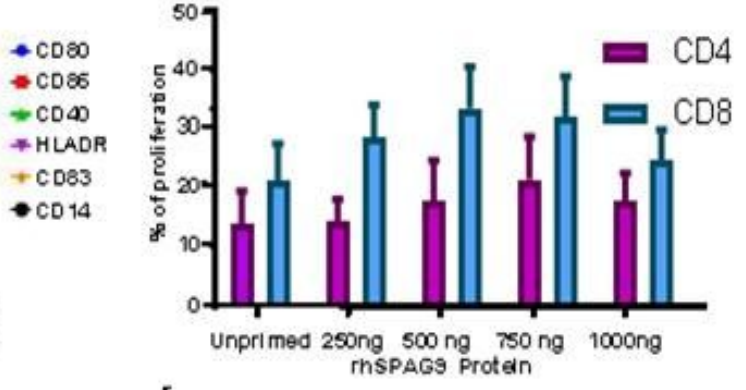

$\mathbf{f}$

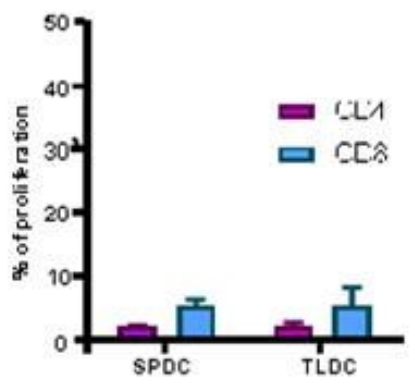

Figure 2

Optimization of rhSPAG9 antigen concentration for priming DCs (a) Percentage of mature DC yield at different concentrations of rhSPAG9 priming (b) Comparative characterization of rhSPAG9 primed DCs and unprimed DCs phenotype (c) Proliferation of CD4+ and CD8+ allogenic PBMCs at various concentrations of rhSPAG9 primed DCs as compared to unprimed DCs. (d) Comparison of Phenotypic characteristics of SPDCs and TLDCs (e) Overall proliferation of responded allogenic PBMCs to SPDCs and TLDCs ( $f$ ) Phenotypic characterization of responded allogenic PBMCs to SPDCs and TLDCs (g) Secretion of IFNy by allogenic PBMCs upon mature DCs stimulation. For figure (a), (b) and (c), data shown is summary of five different experiments (P1-P5). For figure (d), (e), (f) and (g), data shown is summary of three different experiments (P7-P9). 
Figure 3

a

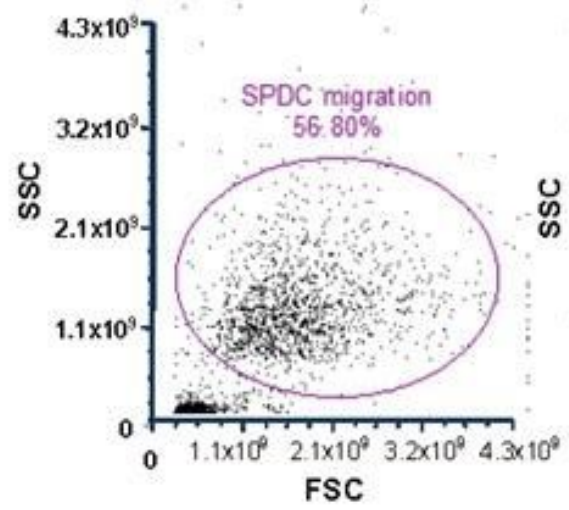

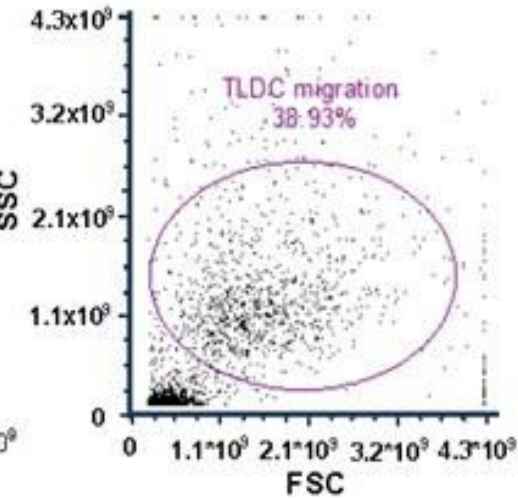

Figure 3

Migration of SPDCs in response to CCL19 and CCL21 (a) Dot plots representing the migratory ability of SPDCs and TLDCs towards the chemokines (b) Box and whisker plot shows data on the migratory capacity of SPDCs and TLDCs towards the chemokine pair (n=3; P7-P9). 
Figure 4
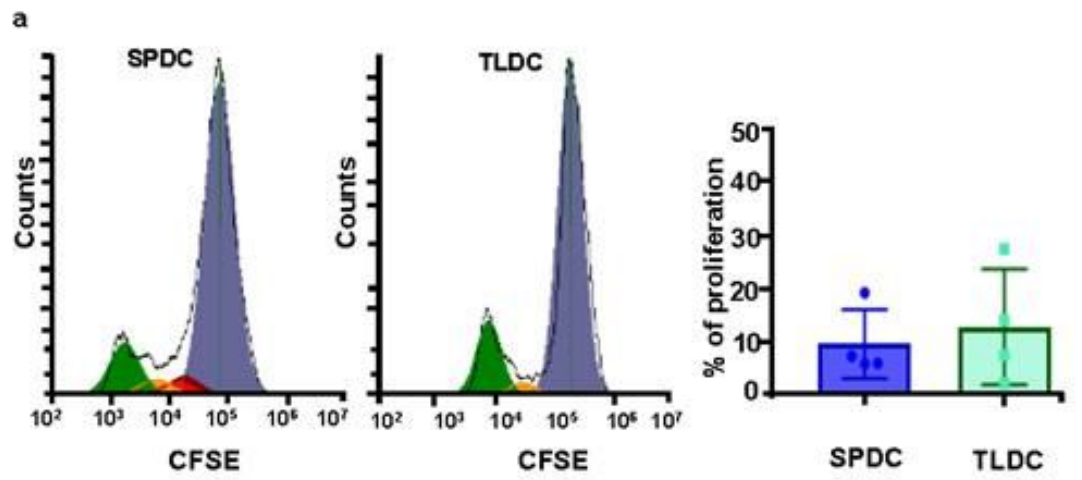

b

c

d
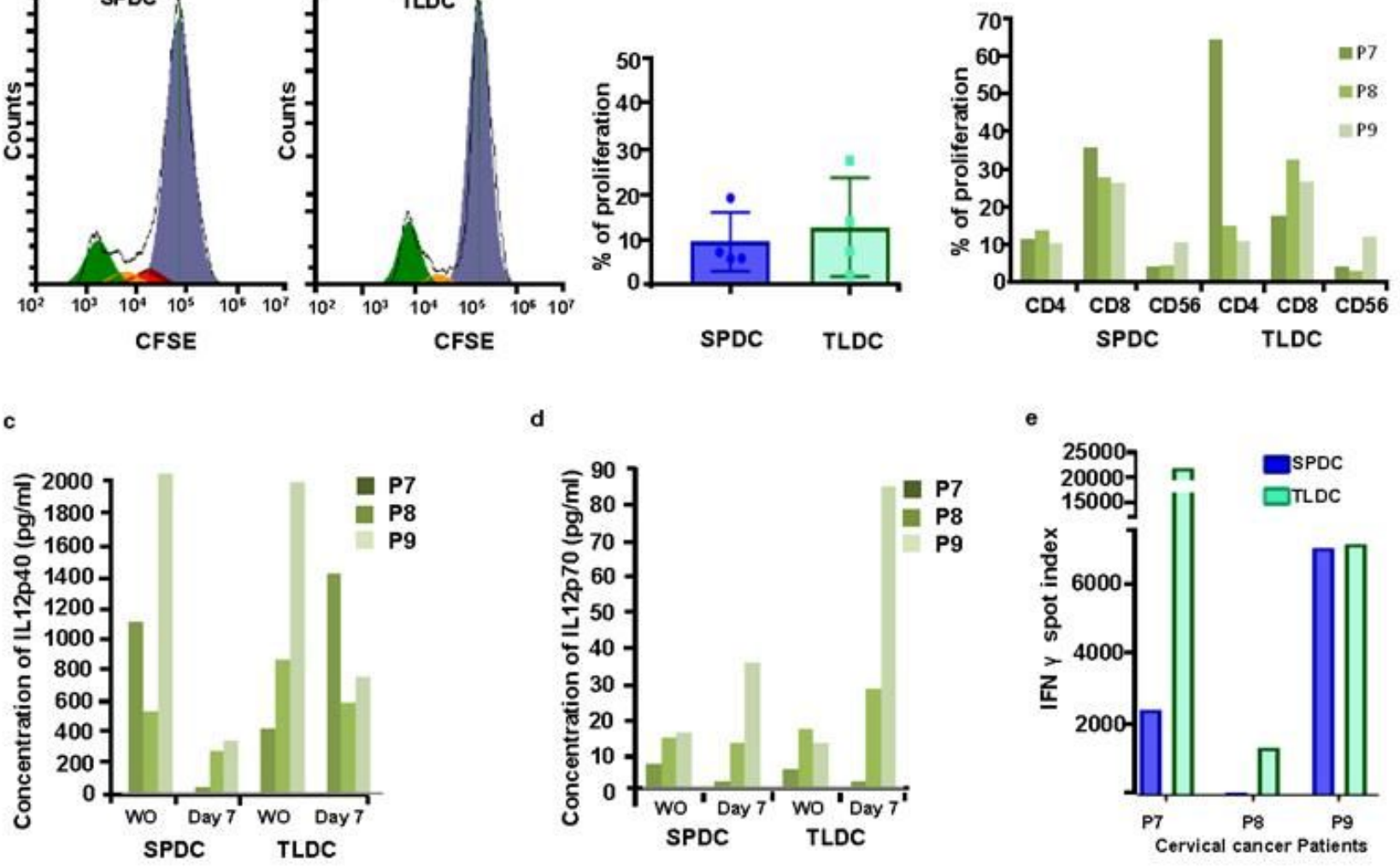

e

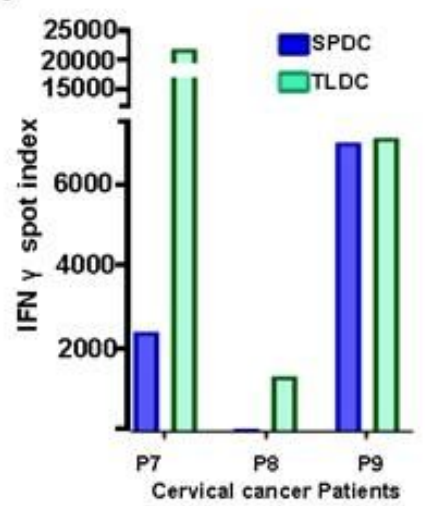

Figure 4

Functional characterization of mature DCs in autologous co-culture (a) Overall proliferation of autologous PBMCs induced in the presence of mature DCs (b) Phenotypic characterization of responding PBMCs upon mature DCs stimulation (c) Data summarizes the level of IL12p40 in wash out and day 7 co-culture supernatants (d) level of IL12p70 in wash out and day 7 co-culture supernatants (e) Secretion of IFNy by autologous PBMCs upon mature DCs stimulation after 14 days of culture. All individual experiments were done in duplicates and Data shown is from three different patients for all the experiments (patient number P7-P9). 
Figure 5

a

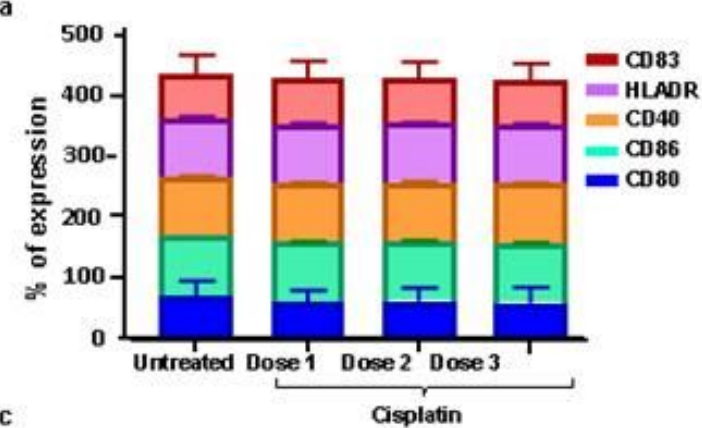

c
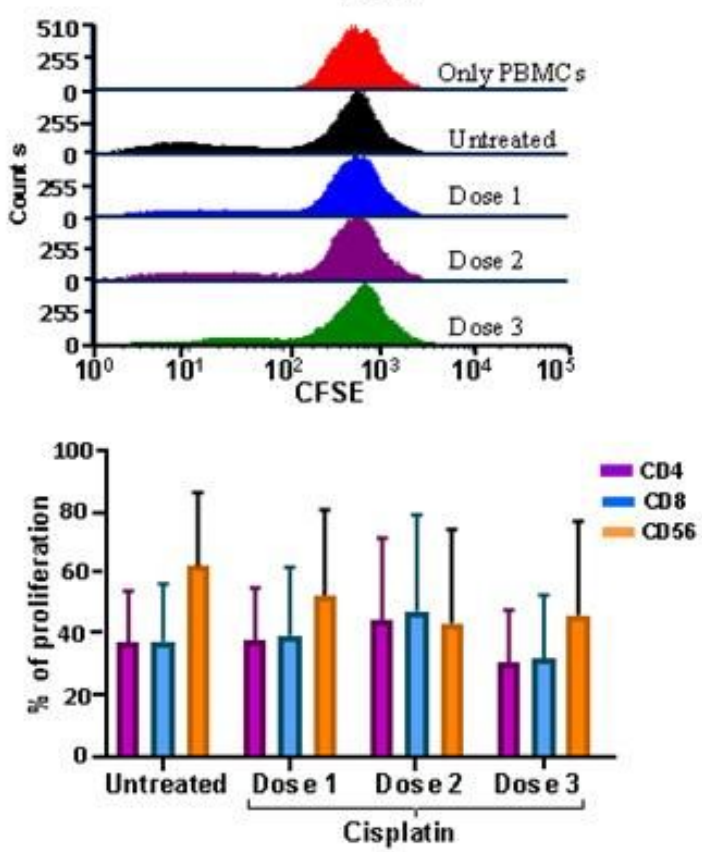

b
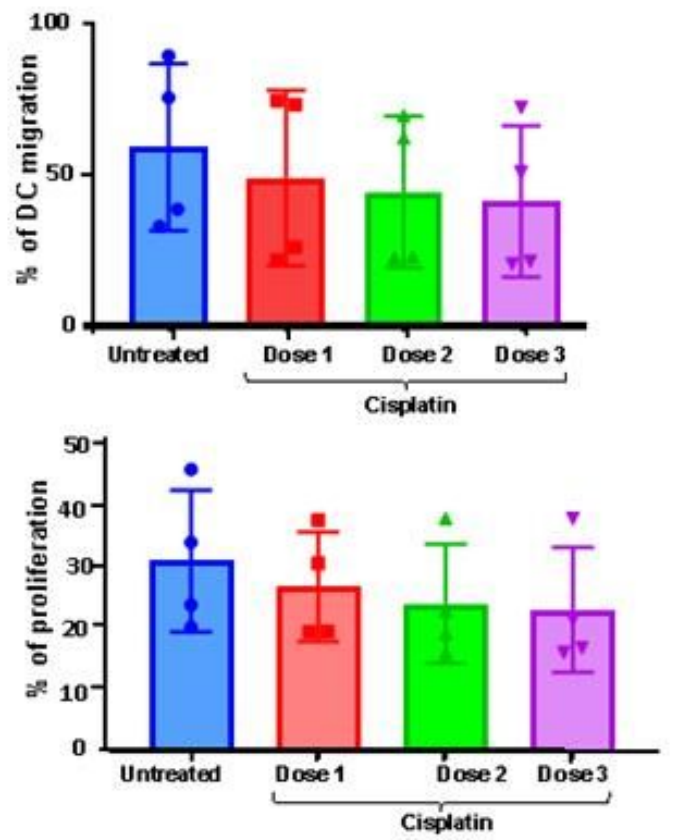

\section{Figure 5}

Characterization of SPDCs in the presence of chemotherapeutic agent cisplatin at three different doses $(150,200$ and $400 \mu \mathrm{M} / \mathrm{ml})$. (a) Phenotypic characterization of SPDCs in the presence or absence of cisplatin (b) Evaluation of migratory capacity of SPDCs with or without the cisplatin in culture medium (c) Data represents the overall proliferation of autologous PBMCs in response to SPDCs in the presence or absence of cisplatin. (d) Overall proliferation of autologous PBMCs in response to SPDCs in the presence or absence of cisplatin (e) Phenotypic characterization of autologous proliferated PBMCs in response to SPDCs. Data summary of three different patients (patient number P10-P12). 
Figure 6
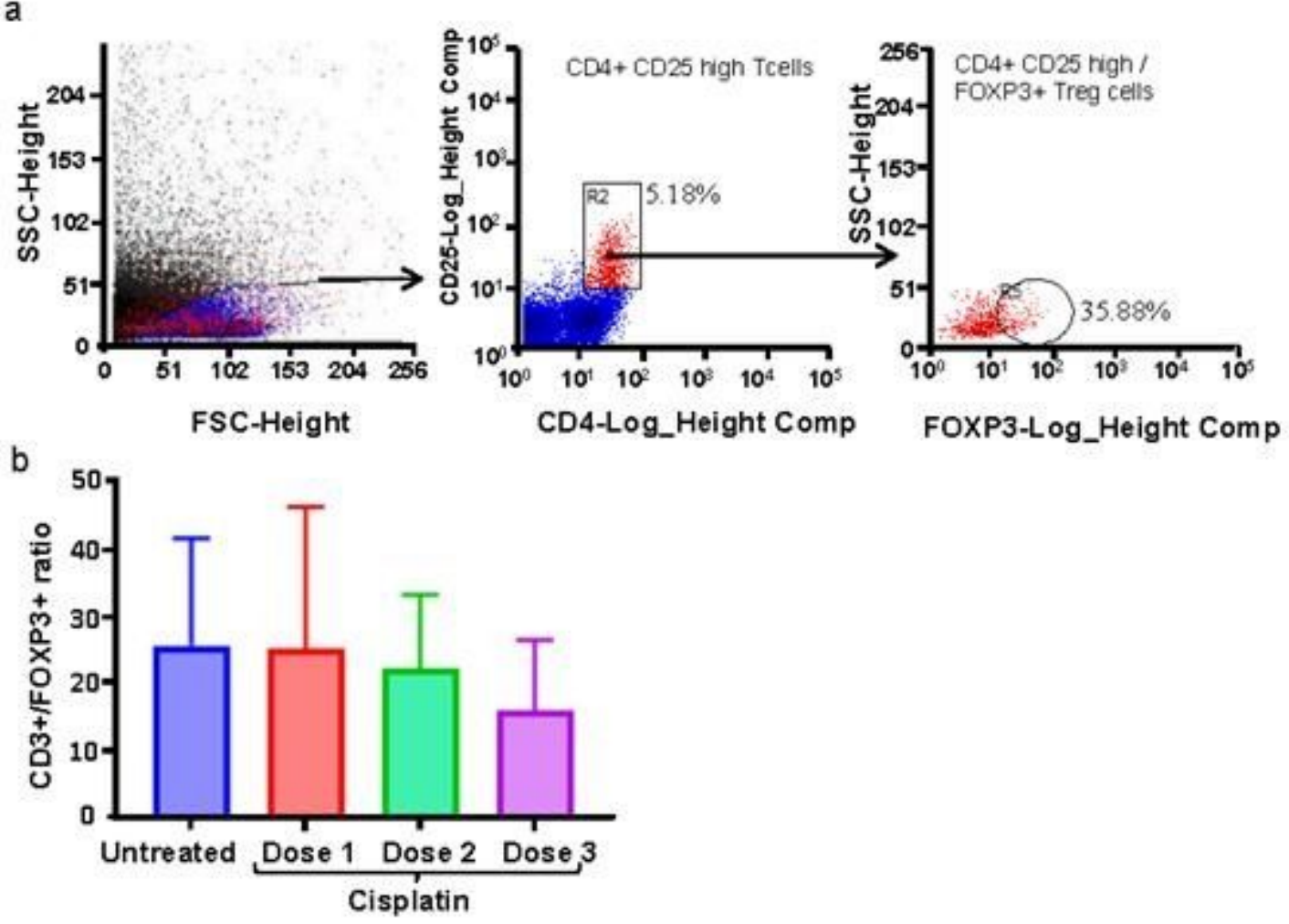

Figure 6

FACS analysis of CD3+/FOXP3+ Tregs in (a) SPDC stimulated co-cultures in the absence of cisplatin (b) Bar graphs indicate $\mathrm{CD} 3+/$ FOXP3+ Tregs ratio in SPDC stimulated co-cultures treated with three different doses of cisplatin treated SPDCs (n=3; P10-P12). 